\title{
Impact of polymerization and storage on the degree of conversion and mechanical properties of veneering resin composites
}

\author{
Felicitas MAYINGER ${ }^{1}$, Marcel REYMUS ${ }^{2}$, Anja LIEBERMANN¹, Marc RICHTER ${ }^{3}$, Patrick KUBRYK ${ }^{3}$, \\ Henning GROßEKAPPENBERG ${ }^{4}$ and Bogna STAWARCZYK ${ }^{1}$ \\ ${ }^{1}$ Department of Prosthetic Dentistry, University Hospital, LMU Munich, Goethestraße 70, 80336 Munich, Germany \\ ${ }^{2}$ Department of Conservative Dentistry and Periodontology, University Hospital, LMU Munich, Goethestraße 70, 80336 Munich, Germany \\ ${ }^{3}$ Spectroscopy Products Division, Renishaw GmbH, Karl-Benz-Strasse 12, 72124 Pliezhausen, Germany \\ ${ }^{4}$ Private Office, Scharnhorstraße 24, 28211 Bremen, Germany \\ Corresponding author, Felicitas MAYINGER; E-mail: felicitas.mayinger@med.uni-muenchen.de
}

\begin{abstract}
To investigate the degree of conversion (DC), Martens hardness (HM), elastic indentation modulus ( $\mathrm{E}_{\text {Iт }}$ ), and flexural strength (FS) of veneering resin composites (SR Nexco Paste (NP), Ceramage Incisal (CI), Gradia Plus (GP); $n=60$ /group) cured with different polymerization devices (bre.Lux Power Unit, Labolight DUO, Otoflash G171, LC-3DPrint Box, PCU LED; $n=12 /$ subgroup) after storage. Otoflash G171 and Labolight DUO showed increased DC/HM/E $\mathrm{E}_{\text {Iт. }}$ CI presented the lowest DC and highest HM/E Iт. NP showed the highest DC and lowest HM/E Іт. Within Otoflash G171, Laboligth DUO and PCU LED, highest FS was observed for CI. Storage did not affect DC/HM/E $\mathrm{E}_{\mathrm{IT}}$ for specimens cured with Otoflash G171 or Labolight DUO. With storage not showing an influence on the tested parameters for polymerization devices that otherwise presented superior results, increased storage time cannot be recommended. For the tested resin composites, this study observed a high/low degree of conversion to coincide with respectively low/ high amounts of fillers/mechanical properties.
\end{abstract}

Keywords: Degree of conversion, Martens hardness, Elastic indentation modulus, Polymerization device, Veneering resin composite

\section{INTRODUCTION}

Resin composites have been firmly established as a restorative material in dentistry for several decades. While being most commonly composed of dimethacrylate monomers such as bisphenol A-glycidyl methacrylate (Bis-GMA), triethyleneglycol dimethacrylate (TEGDMA) or urethane dimethacrylate (UDMA), fillers (e.g. silica) and a photoinitiator, resin composites vary in regards to the filler size and can thus be classified as macro-, micro-, hybrid-, nano- or bulk filled, each representing different advantages and disadvantages regarding flexural strength, wear resistance, and surface quality ${ }^{1)}$. While microfilled resin composites with a particle size of about $0.4 \mu \mathrm{m}$ are easy to polish, the decreased overall filler load of only $40-45 \%$ impairs mechanical properties ${ }^{2}$. This shortcoming was addressed by the development of nanoparticles $(20-70 \mathrm{~nm})$, that have been observed to form nanocluster units, resulting in a high mechanical strength similar to hybrid materials composed of both macro- and microfilled particles ${ }^{3,4}$. Nanofilled resin composites have however been described to pose difficulties when being adapted into small spaces, as the high filler volume decreases formability ${ }^{4}$. In contrast to direct resin composites employed by a dentist for fillings, indirect resin composites are used in dental laboratories for a wide range of settings, from temporary restorations of all sorts, to veneers, in- or onlays, implant-supported removable dentures or as veneering

Color figures can be viewed in the online issue, which is available at J-STAGE.

Received Nov 21, 2019: Accepted Jun 15, 2020

doi:10.4012/dmj.2019-394 JOI JST.JSTAGE/dmj/2019-394 material for fixed dental prostheses. With indirect resin composites containing an increased volume of fillers and the extraoral setting enabling an increased curing time, these materials can provide enhanced mechanical properties ${ }^{5,6)}$.

One factor of utmost importance for a resin composite's structural stability, but above all its biocompatibility, is the achieved degree of conversion (i.e. the percentage of vinyl functions converted to aliphatic functions) after undergoing polymerization ${ }^{7,8}$. Composites are cured in a chemical reaction called chain polymerization, which can be started by thermal-, high energy- or chemical initiation. Chain carriers (e.g. free radicals) initiate the polymerization and lead to a conversion of double to single bonds, thus linking monomers to form polymers. High energy initiation, which is the most common curing method used for resin composites, is accomplished by irradiation employing different polymerization devices: from halogen to lightemitting diodes (LEDs) emitting light in a constant or intermittent setting (e.g. flashlights) at varying wavelength spectra. The wavelength of a polymerization device is set to match the photoinitiation system of the respective material, with the principal photoinitiator used in contemporary single-component resin composite pastes being camphorquinone (maximum absorption at $468 \mathrm{~nm}$ ), paired with a coinitiator (e.g. a tertiary amine) $)^{9}$. As camphorquinone can introduce a yellow color shade to the composite, impairing the esthetic appearance, alternatives such as phenylpropanedione (maximum absorption at $398 \mathrm{~nm}$ ) have been sought out ${ }^{10)}$. In ideal 
conditions, chain propagation, in this case triggered by photoinitiation, would continue until all monomers have converted to a polymer network. Light curing however only enables a material's polymerization to a certain depth, dependent on many factors from the type of resin composite to the polymerization device and irradiation time. When in contact with solvents (e.g. saliva), unbound monomers can be eluted and incorporated into the circulatory system, causing systemic responses from allergies to teratogenic or even carcinogenic effects $^{11,12)}$. It is thus vital to ensure a high degree of conversion and in consequence minimal percentage of unpolymerized monomers. While various test methods, from gas chromatography/mass spectrometry, infrared spectroscopy, ultraviolet-visible spectrophotometry to nuclear magnetic resonance spectroscopy, exist, each method entails its own limitations in regards to costs and time, but more importantly reliability and accuracy. One relatively unknown approach in the field of dental material science is the use of Raman spectroscopy to determine vibration bands, thus enabling inferences on a material's chemical composition ${ }^{13)}$. With $\mathrm{C}=\mathrm{C}$ double bonds converting to $\mathrm{C}$-C single bonds in a free radical polymerization reaction, a material's degree of conversion can be determined by comparing the vibration bands of the residual unpolymerized methacrylate $\mathrm{C}=\mathrm{C}$ stretching mode at $1,640 \mathrm{~cm}^{-1}$ to the aromatic $\mathrm{C}=\mathrm{C}$ stretching mode at $1,610 \mathrm{~cm}^{-1}$ used as an internal standard before and after polymerization ${ }^{14)}$. This test method thus enables a comparison of the influence of the use of different resin composites and polymerization devices on the degree of conversion.
This relatively unknown approach has been employed in few previous examinations in the field of dental resin composites ${ }^{14,15)}$. To the authors' best knowledge, veneering resin composites have not been examined in this regard. Standard examination procedures such as the determination of Martens hardness and elastic indentation modulus to assess a material's mechanical properties directly after polymerization and in the course of several time intervals were thus incorporated in this novel study-design to enable the critical assessment of the use of Raman spectroscopy in this context, as a high degree of conversion should correlate with superior mechanical properties ${ }^{16)}$.

The aim of this investigation was to examine the degree of conversion, Martens hardness, elastic indentation modulus, and flexural strength of three veneering resin composites cured with five polymerization devices after different storage intervals. The tested hypotheses stated that neither the use of different veneering resin composites, nor the choice of polymerization device or storage interval showed an impact on the degree of conversion, Martens hardness, elastic indentation modulus or flexural strength.

\section{MATERIALS AND METHODS}

Three veneering resin composites were examined: SR Nexco Paste (NP, Incisal I3, Ivoclar Vivadent, Schaan, Liechtenstein), Ceramage Incisal (CI, 59, SHOFU, Kyoto, Japan) and Gradia Plus (GP, HB-ED, GC, Tokyo, Japan) (Fig. 1, Table 1).

Veneering resin composites
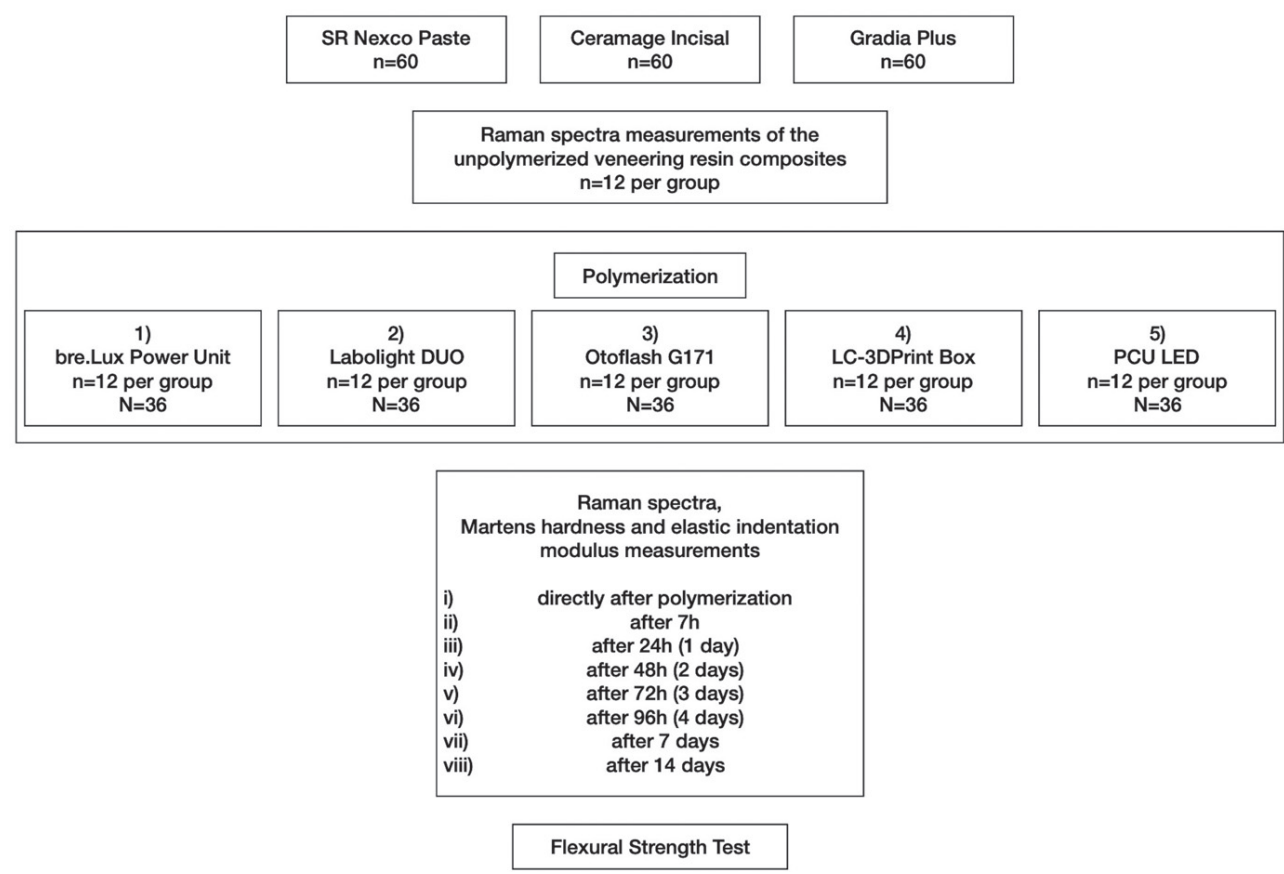

Fig. 1 Study design. 
Table 1 Materials, abbreviations, manufacturers, type of veneering resin composite, compositions and Lot.No. used

\begin{tabular}{|c|c|c|c|c|c|}
\hline Material & Abbreviation & Manufacturer & $\begin{array}{l}\text { Type of } \\
\text { veneering resin } \\
\text { composite }\end{array}$ & Composition & Lot.No. \\
\hline $\begin{array}{l}\text { SR Nexco } \\
\text { Paste } \\
\text { (Incisal I3) }\end{array}$ & NP & $\begin{array}{l}\text { Ivoclar Vivadent, } \\
\text { Schaan, } \\
\text { Liechtenstein }\end{array}$ & Nanohybrid & $\begin{array}{l}\text { 17-19 wt\%: dimethacrylates, } \\
82-83 \mathrm{wt} \% \text { : copolymer and silicon } \\
\text { dioxide, <1 wt\%: stabilizers, catalysts } \\
\text { and pigments, } \\
64-65 \text { wt\%: total content of inorganic } \\
\text { fillers (particle size } 10-100 \mathrm{~nm} \text { ) }\end{array}$ & W04319 \\
\hline $\begin{array}{l}\text { Ceramage } \\
\text { Incisal (59) }\end{array}$ & $\mathrm{CI}$ & $\begin{array}{l}\text { SHOFU, Kyoto, } \\
\text { Japan }\end{array}$ & Microhybrid & $\begin{array}{l}\text { 5-15\%: urethane dimethacrylate, } \\
\text { urethane diacrylate, } \\
73 \mathrm{wt} \% \text { : zirconium silicate filler, } \\
\text { pigments and others }\end{array}$ & 101671 \\
\hline $\begin{array}{l}\text { Gradia Plus } \\
\text { (HB-ED) }\end{array}$ & GP & GC, Tokyo, Japan & Nanohybrid & $\begin{array}{l}\text { 1-5\%: bisphenol A-glycidyl methacrylate, } \\
\text { 5-10\%: triethylene glycol dimethacrylate, } \\
\text { 1-5\%: urethane dimethacrylate, } \\
\text { ceramic filler }\end{array}$ & 1703131 \\
\hline
\end{tabular}

\section{Specimen preparation}

Specimens were manufactured to form round disks using a hollow acrylonitrile butadiene styrene form (SD Mechatronik, Feldkirchen-Westerham, Germany), into which the veneering resin composites were pressed manually ( $n=60$ /group, $\mathrm{N}=180$ ). A glass microscope slide isolated with petroleum jelly (Vaselinum, Fagron, Glinde, Germany) was placed on top of each specimen prior to polymerization to ensure a smooth surface. Residual composite was removed after polymerization using a Le Cron Instrument. Final specimen dimensions were set to $12 \times 1.5 \mathrm{~mm}$. Subsequently, specimens were cured with five different polymerization devices $(n=12 /$ subgroup):

1) bre.Lux Power Unit (bredent, Senden, Germany) for $180 \mathrm{~s}$ with twenty-one LEDs at a wavelength of $370-500 \mathrm{~nm}$.

2) Labolight DUO (GC Europe, Leuven, Belgium) for $180 \mathrm{~s}$ with twelve blue and three purple LEDs at a wavelength of $380-510 \mathrm{~nm}$ with approximate peaks at 395 and $475 \mathrm{~nm}$.

3) Otoflash G171 (NK Optik, Baierbrunn, Germany) under nitrogen atmosphere for 1.800 flashes using two flashlight lamps at a wavelength of 280-580 $\mathrm{nm}$ with approximate peaks at 480 and $530 \mathrm{~nm}$ and a light intensity of approximately $1.4 \mathrm{~W} / \mathrm{cm}^{2}$ per flash.

4) LC-3DPrint Box (NextDent, Soesterberg, Netherlands) for $180 \mathrm{~s}$ with six $1.7 \mathrm{~W}$ ultra-violet light (UV) lamps and six $2.3 \mathrm{~W}$ UV lamps at a wavelength of $315-550 \mathrm{~nm}$ with approximate peaks at 360 and $435 \mathrm{~nm}$.

5) PCU LED (Dreve, Unna, Germany) under vacuum for $180 \mathrm{~s}$ with eighteen LED lamps at $80 \%$ power at a wavelength of $410 \mathrm{~nm}$ and a light intensity of $1.4 \mathrm{~mW} / \mathrm{cm}^{2}$.

To remove any surplus petroleum jelly, each specimen's surface was cleaned with a paper wipe soaked in 96\% ethanol (Otto Fischar, Saarbrücken, Germany) before being stored at room temperature $\left(23^{\circ} \mathrm{C}\right)$ under natural light.

\section{Raman spectra}

Raman spectra were acquired on a confocal Raman spectrophotometer (inVia Qontor, Renishaw, New Mills, UK). Unpolymerized veneering resin composite was applied to a microscope slide and examined to determine the Raman scattering of the unpolymerized material $\left(R_{\text {unpolymerized }}\right)$. Twelve initial measurements for each veneering resin composite were performed to compute an average value for $R_{\text {unpolymerized }}$. After polymerization, cured specimens were examined to determine $R_{\text {cured }}$ after different storage intervals: i) directly after polymerization, ii) after $7 \mathrm{~h}$, iii) after $24 \mathrm{~h}$ (1 day), iv) after 48 h (2 days), v) after 72 h (3 days), vi) after 96 h (4 days), vii) after 7 days and viii) after 14 days. Specimens were excited by a diode laser at a wavelength of $785 \mathrm{~nm}$ and a spectral resolution of $1 \mathrm{~cm}^{-1}$ through a microscope objective (50×). Three accumulations were conducted for each measurement at a power of $100 \%$ and an irradiation time of $10 \mathrm{~s}$. The resulting Raman spectra were analyzed with the software WiRE 4.2 (Renishaw) in a spectral range of 2,000-1,500 $\mathrm{cm}^{-1}$. Band height was recorded at peaks $1,640 \mathrm{~cm}^{-1}$ ((un)polymerized methacrylate $\mathrm{C}=\mathrm{C}$ stretching mode) and $1,610 \mathrm{~cm}^{-1}$ (aromatic $\mathrm{C}=\mathrm{C}$ stretching mode) after using the integrated Curve Fit function. The degree of conversion (DC) was calculated using the following formula:

$$
\begin{aligned}
& D C(\%)=100 *\left[1-\frac{R_{\text {cured }}}{R_{\text {unpolymerized }}}\right], \\
& \text { where } R=\frac{\text { band height at } 1640 \mathrm{~cm}^{-1}}{\text { band height at } 1610 \mathrm{~cm}^{-1}} .
\end{aligned}
$$




\section{Martens hardness and elastic indentation modulus} Martens hardness (HM) and elastic indentation modulus $\left(E_{I T}\right)$ were assessed at each storage interval using an universal hardness testing machine (Zwick/Roell ZHU 0.2, Zwick, Ulm, Germany). For this purpose, the diamond indenter pyramid of the machine was vertically pressed into the specimens' surface with a load of 9.8 $\mathrm{N}$ for $2 \mathrm{~s}$. All specimens were tested at three different points and $\mathrm{HM}$ and $\mathrm{E}_{\mathrm{IT}}$ values were calculated (TestXpert v.12.3 Master; Zwick) with the following equations ${ }^{17)}$ :

$$
H M=\frac{F}{A_{S}(h)}
$$

where $H M$ is Martens hardness $\left(\mathrm{N} / \mathrm{mm}^{2}\right) ; F$ is test force $(\mathrm{N}) ; A_{S}(h)$ is surface area of the indenter at distance $h$ from the tip $\left(\mathrm{mm}^{2}\right)$, and

$$
E_{I T}=\left(1-v_{s}^{2}\right)\left(\frac{2 \sqrt{A_{p}\left(h_{c}\right)}}{\sqrt{\pi S}}-\frac{\left(1-v_{i}^{2}\right)}{E_{i}}\right)^{-1}
$$

where $E_{I T}$ is the elastic indentation modulus of the indenter (Vickers pyramid) $\left(\mathrm{kN} / \mathrm{mm}^{2}\right), v_{s}$ is Poisson's ratio of the specimen $\left(v_{s}=0.3\right)^{18)}$ and $v_{i}$ is Poisson's ratio of the indenter $\left(v_{i}=0.3\right)$.

\section{Flexural strength}

Biaxial strength was tested according to DIN EN ISO $6872: 2008^{19)}$ at room temperature $\left(23^{\circ} \mathrm{C}\right)$. The diameter of each specimen was previously determined with IP65 digital micrometer screw (Mitutoyo Deutschland, Neuss, Germany) to a precision of $0.01 \mathrm{~mm}$. Specimens were positioned in the sample holder of the Universal Testing Machine (Zwick Z010, Zwick), comprised of three tempered steel balls with a diameter of $3.2 \mathrm{~mm}$ forming an equilateral triangle with an edge length of 10 $\mathrm{mm}$ and a ball support circle of $120^{\circ}$ (Fig. 2). The center of the tested specimens and the center of the equilateral

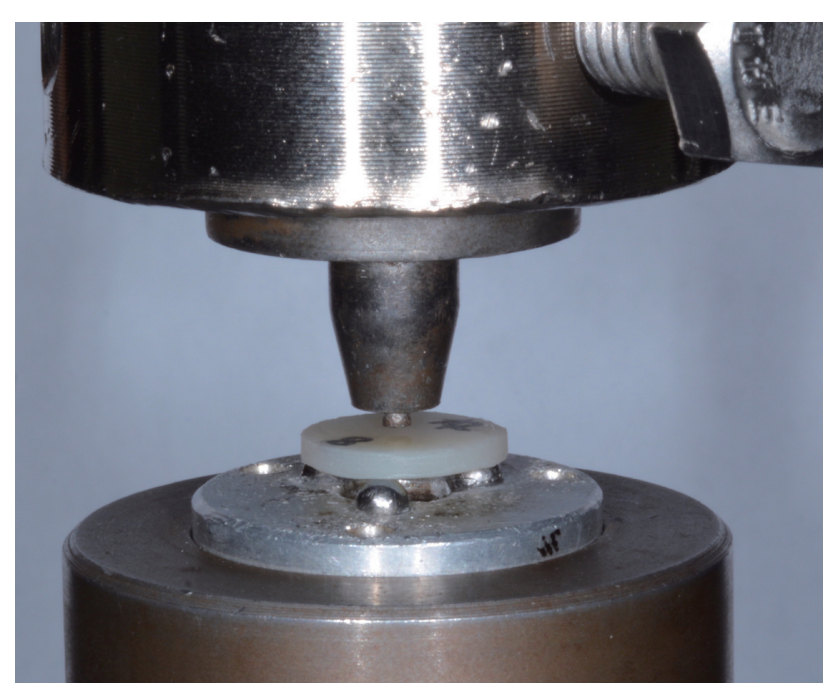

Fig. 2 Flexural strength test. triangle were aligned coaxially. Crosshead speed was set to $1 \mathrm{~mm} / \mathrm{min}$ and force was applied from above with a plunger of $1.4 \mathrm{~mm}$ diameter until failure. Flexural strength (FS) was calculated according to the formula:

$$
\sigma=-0.25 N^{*}(X-Y) / d^{2}
$$

where $\sigma$ is flexural strength, $N$ is fracture load and coefficients $X$ and $Y$ represent:

$$
\begin{aligned}
& X=(1+v) \ln \left[\left(r_{2} / r_{3}\right)\right]^{2}+[(1-v) / 2]\left(r_{2} / r_{3}\right)^{2} \\
& Y=(1+v)\left[1+\ln \left[\left(r_{1} / r_{3}\right)^{2}\right]+(1-v)\left(r_{1} / r_{3}\right)^{2}\right.
\end{aligned}
$$

where $v$ is Poisson's ratio $(v=0.3) ; r_{1}$ is mean support contact diameter $(\mathrm{mm}) ; r_{2}$ is mean loaded contact diameter $(\mathrm{mm}) ; r_{3}$ is diameter of the specimen $(\mathrm{mm})$ and $d$ is thickness of the specimen ( $\mathrm{mm})$.

\section{Statistical analysis}

Statistical evaluation of the results was performed with descriptive analysis followed by Kolmogorov-Smirnov for testing the violation of normal distribution. For global analysis, univariate ANOVAs and partial eta squared $\left(\eta_{\mathrm{p}}^{2}\right)$ were calculated. Furthermore, Kruskal-Wallis-, Mann-Whitney-U-, Wilcoxon-, and Friedman-test were computed to analyze significant differences between tested groups. Additionally, the Weibull distribution parameter (Weibull modulus) was calculated using the maximum likelihood estimation method and $95 \%$ confidence interval ${ }^{20)}$. All $p$-values below 0.05 were construed as statistically significant, with the exception of $\mathrm{DC}, \mathrm{HM}$, and $\mathrm{E}_{\mathrm{IT}}$ results analyzed in regard to the influence of storage, which were adjusted by Bonferroni correction (DC: $p<0.0085$, HM: $p<0.000125$ and $\mathrm{E}_{\mathrm{IT}}$ : $p=0.000125)$. Data were analyzed with SPSS version 25.0 (IBM, Armonk, NY, USA).

\section{RESULTS}

The results of the descriptive statistics are presented in Tables 2, 3 and 4. As the Kolmogorov-Smirnov test indicated a violation of the assumption of normality for $13 \%$ of the tested FS, $10 \%$ of the tested DC, $28 \%$ of the tested HM, and $19 \%$ of the tested $\mathrm{E}_{\mathrm{IT}}$ groups, nonparametric tests were performed. The veneering resin composite exerted the highest influence $(p<0.001$; DC: partial eta squared $\eta_{\mathrm{p}}{ }^{2}=0.666 ; \mathrm{HM}: \eta_{\mathrm{p}}{ }^{2}=0.359 ; \mathrm{E}_{\mathrm{IT}}$ $\left.\eta_{\mathrm{p}}{ }^{2}=0.562 ; \mathrm{FS} \eta_{\mathrm{p}}{ }^{2}=0.286\right)$, followed by the polymerization device $\left(p<0.001\right.$ for DC: $\eta_{\mathrm{p}}{ }^{2}=0.497$; H: $\eta_{\mathrm{p}}{ }^{2}=0.271$ and $\mathrm{E}_{\mathrm{TT}}$ : $\eta_{\mathrm{p}}{ }^{2}=0.215$; FS: $\left.p=0.004, \eta_{\mathrm{p}}{ }^{2}=0.088\right)$ and storage interval $\left(p<0.001 ;\right.$ HM: $\left.\eta_{\mathrm{p}}{ }^{2}=0.119 ; \mathrm{E}_{\mathrm{IT}}: \eta_{\mathrm{p}}{ }^{2}=0.213\right)$. The effect of the binary combination of the parameters veneering resin composite and polymerization device $(p<0.001$ for DC: $\eta_{\mathrm{p}}{ }^{2}=0.151$; HM: $\eta_{\mathrm{p}}{ }^{2}=0.064$ and $\mathrm{E}_{\mathrm{IT}}: \eta_{\mathrm{p}}{ }^{2}=0.028$; FS: $\left.p=0.002, \eta_{\mathrm{p}}{ }^{2}=0.138\right)$, veneering resin composite and storage interval (HM: $p<0.001, \eta_{\mathrm{p}}{ }^{2}=0.030$; $\mathrm{E}_{\mathrm{IT}}: p=0.007$, $\eta_{\mathrm{p}}{ }^{2}=0.023$ ), and polymerization device and storage interval was also significant (HM: $p<0.005, \eta_{\mathrm{p}}{ }^{2}=0.037$; $\left.\mathrm{E}_{\mathrm{IT}}: p<0.001, \eta_{\mathrm{p}}{ }^{2}=0.059\right)$. The ternary combination of the three parameters had an effect on $\mathrm{E}_{\mathrm{IT}}(p=0.007$, 
Table 2 Degree of conversion for all tested groups

\begin{tabular}{|c|c|c|c|c|c|}
\hline \multirow[t]{2}{*}{$\mathrm{DC}$} & bre.Lux Power Unit & Labolight DUO & Otoflash G171 & LC-3DPrint Box & PCU LED \\
\hline & Mean \pm SD & Mean \pm SD & Mean \pm SD & Mean \pm SD & Mean \pm SD \\
\hline \multicolumn{6}{|l|}{ i) } \\
\hline NP & $51.5 \pm 1.96^{\mathrm{cA} \alpha}$ & $55.4 \pm 3.30^{\mathrm{cB} \alpha}$ & $62.3 \pm 3.33^{\mathrm{cC} \alpha}$ & $53.6 \pm 4.20^{\mathrm{cAB} \alpha}$ & $52.9 \pm 1.38^{\mathrm{cAB} \alpha}$ \\
\hline CI & $39.1 \pm 2.38^{* \mathrm{aB} \alpha}$ & $39.7 \pm 8.49 * \mathrm{aB} \alpha$ & $52.8 \pm 2.65^{\mathrm{aC} \alpha}$ & $30.7 \pm 10.4^{\mathrm{aA \alpha} \alpha}$ & $32.2 \pm 3.16^{\mathrm{aA} \alpha}$ \\
\hline GP & $49.0 \pm 1.16^{\mathrm{bC} \alpha \beta}$ & $52.1 \pm 1.94^{\mathrm{bC} a}$ & $57.9 \pm 2.07^{\mathrm{bD} \alpha}$ & $46.5 \pm 3.30^{\mathrm{bA} \alpha}$ & $48.8 \pm 2.10^{\mathrm{bAB} \alpha}$ \\
\hline \multicolumn{6}{|l|}{ ii) } \\
\hline NP & $50.8 \pm 3.50^{\mathrm{bA \alpha} \alpha}$ & $56.0 \pm 2.49^{\mathrm{cC} \alpha}$ & $61.8 \pm 3.98^{\mathrm{cD} \alpha}$ & $52.5 \pm 5.11^{* \mathrm{cAB} a}$ & $54.1 \pm 1.92^{\mathrm{cB} \alpha}$ \\
\hline $\mathrm{CI}$ & $38.5 \pm 2.90^{\mathrm{aB} \alpha}$ & $39.2 \pm 17.9 * \mathrm{aC} a$ & $50.4 \pm 4.20 * \mathrm{aD} \alpha$ & $30.6 \pm 8.93^{\mathrm{aA \alpha}}$ & $32.5 \pm 7.38^{\mathrm{aA} \alpha}$ \\
\hline GP & $47.8 \pm 3.07^{\mathrm{bA} \alpha}$ & $54.3 \pm 3.28^{* \mathrm{bB} \alpha}$ & $57.2 \pm 2.61^{\mathrm{bC} \alpha}$ & $47.0 \pm 2.40^{\mathrm{bA} \alpha}$ & $49.0 \pm 2.27^{\mathrm{bA} \alpha}$ \\
\hline \multicolumn{6}{|l|}{ iii) } \\
\hline NP & $52.7 \pm 4.31^{\mathrm{cA} \alpha}$ & $56.3 \pm 1.84^{\mathrm{bB} \alpha}$ & $63.5 \pm 2.90^{\mathrm{cC} \alpha}$ & $52.3 \pm 3.96^{\mathrm{cA \alpha}}$ & $54.8 \pm 3.14^{\mathrm{cAB} \alpha}$ \\
\hline $\mathrm{CI}$ & $40.0 \pm 4.13^{\mathrm{aB} \alpha}$ & $43.9 \pm 4.87^{* \mathrm{aC} a}$ & $50.7 \pm 4.68^{\mathrm{aD} \alpha}$ & $32.8 \pm 6.63^{\mathrm{aA \alpha}}$ & $33.0 \pm 4.59^{\mathrm{aA} \alpha}$ \\
\hline GP & $48.4 \pm 3.17^{\mathrm{bAB} \alpha \beta}$ & $54.9 \pm 2.64^{\mathrm{bC} \alpha}$ & $56.7 \pm 3.59^{\mathrm{bC} \alpha}$ & $45.9 \pm 3.26^{\mathrm{bA \alpha}}$ & $50.0 \pm 3.04^{\mathrm{bB} \alpha}$ \\
\hline \multicolumn{6}{|l|}{ iv) } \\
\hline NP & $51.7 \pm 4.18^{\mathrm{bA \alpha}}$ & $56.7 \pm 3.36^{\mathrm{bB} \alpha}$ & $63.5 \pm 2.05^{\mathrm{cC} \alpha}$ & $55.6 \pm 3.42^{\mathrm{cB} \alpha}$ & $55.1 \pm 2.71^{\mathrm{cB} \alpha}$ \\
\hline CI & $38.5 \pm 4.23^{\mathrm{aB} \alpha}$ & $44.7 \pm 3.47^{\mathrm{aC} a}$ & $50.3 \pm 5.01^{\mathrm{aD} \alpha}$ & $32.0 \pm 7.31^{\mathrm{aA} \alpha}$ & $32.9 \pm 5.65^{* \mathrm{aA} \alpha}$ \\
\hline GP & $49.7 \pm 2.55^{\mathrm{bB} \alpha \beta}$ & $53.9 \pm 3.46^{\mathrm{bC} a}$ & $58.7 \pm 2.12^{\mathrm{bD} \alpha}$ & $45.8 \pm 4.62^{\mathrm{bA} \alpha}$ & $48.5 \pm 2.64^{\mathrm{bAB} \alpha}$ \\
\hline \multicolumn{6}{|l|}{ v) } \\
\hline NP & $51.3 \pm 3.86^{\mathrm{bA} \alpha}$ & $56.1 \pm 3.25^{\mathrm{bB} \alpha}$ & $63.1 \pm 3.72^{\mathrm{cC} \alpha}$ & $52.6 \pm 4.35^{\mathrm{cAB} \alpha}$ & $53.7 \pm 2.91^{\mathrm{cAB} \alpha}$ \\
\hline $\mathrm{CI}$ & $35.8 \pm 5.00^{\mathrm{aA} \alpha}$ & $43.7 \pm 7.23^{* \mathrm{a} \mathrm{B} a}$ & $51.4 \pm 5.10^{\mathrm{aC} \alpha}$ & $31.3 \pm 8.94^{* \mathrm{aA} \alpha}$ & $33.7 \pm 3.02^{\mathrm{aA} \alpha}$ \\
\hline GP & $50.7 \pm 2.68^{\mathrm{bB} \alpha \beta}$ & $54.3 \pm 3.34^{\mathrm{bCa}}$ & $59.5 \pm 3.07^{\mathrm{bD} \alpha}$ & $46.9 \pm 2.70^{\mathrm{bA} \alpha}$ & $48.5 \pm 3.89^{\mathrm{bA} \alpha}$ \\
\hline \multicolumn{6}{|l|}{ vi) } \\
\hline NP & $51.3 \pm 4.68^{\mathrm{bA} \alpha}$ & $56.8 \pm 2.48^{\mathrm{bB} \alpha}$ & $62.7 \pm 3.15^{\mathrm{cC} \alpha}$ & $54.0 \pm 3.88^{\mathrm{cA} a}$ & $54.5 \pm 3.24^{\mathrm{cAB} \alpha}$ \\
\hline $\mathrm{CI}$ & $40.8 \pm 8.72^{\mathrm{aB} \alpha}$ & $46.4 \pm 4.31^{\mathrm{aC} \alpha}$ & $51.7 \pm 5.67^{\mathrm{aD} \alpha}$ & $31.5 \pm 10.0^{* a \mathrm{AB} \alpha}$ & $30.8 \pm 6.44^{\mathrm{aA} \alpha}$ \\
\hline GP & $49.5 \pm .39^{\mathrm{bA \alpha} \beta}$ & $55.6 \pm 3.51^{\mathrm{bB} \alpha}$ & $58.6 \pm 2.94^{\mathrm{bC} \alpha}$ & $46.3 \pm 4.75^{\mathrm{bA \alpha} \alpha}$ & $47.7 \pm 2.48^{\mathrm{bA \alpha} \alpha}$ \\
\hline \multicolumn{6}{|l|}{ vii) } \\
\hline $\mathrm{NP}$ & $52.4 \pm 3.63^{\mathrm{cAB} \alpha}$ & $50.3 \pm 2.49^{\mathrm{bAa} a}$ & $62.5 \pm 3.22^{\mathrm{cC} \alpha}$ & $53.5 \pm 3.97^{\mathrm{cB} \alpha}$ & $53.4 \pm 3.23^{\mathrm{cB} \alpha}$ \\
\hline CI & $37.1 \pm 4.95^{\mathrm{aC} \alpha}$ & $40.4 \pm 11.3^{\mathrm{aC} \alpha}$ & $51.3 \pm 4.38^{\mathrm{aD} \alpha}$ & $33.3 \pm 6.97^{\mathrm{aA} \alpha}$ & $28.3 \pm 3.40^{\mathrm{aB} \alpha}$ \\
\hline GP & $49.0 \pm 2.71^{\mathrm{bAB} \alpha \beta}$ & $53.8 \pm 1.96^{\mathrm{cC} \alpha}$ & $58.2 \pm 2.82^{\mathrm{bD} \alpha}$ & $47.0 \pm 2.53^{\mathrm{bA} \alpha}$ & $49.4 \pm 1.71^{\mathrm{bB} \alpha}$ \\
\hline \multicolumn{6}{|l|}{ viii) } \\
\hline $\mathrm{NP}$ & $47.0 \pm 4.68^{\mathrm{bA} \alpha}$ & $52.5 \pm 6.95^{\mathrm{bB} \alpha}$ & $58.8 \pm 5.18^{\mathrm{bC} \alpha}$ & $52.4 \pm 5.02^{* \mathrm{cB} \alpha}$ & $54.4 \pm 2.31^{\mathrm{cB} \alpha}$ \\
\hline $\mathrm{CI}$ & $38.9 \pm 6.34^{\mathrm{aA} \alpha}$ & $42.1 \pm 11.9^{\mathrm{aAB} \alpha}$ & $47.8 \pm 10.9^{\mathrm{aB} \alpha}$ & $35.4 \pm 8.58^{\mathrm{aA} \alpha}$ & $33.5 \pm 10.6^{\mathrm{aA} \alpha}$ \\
\hline GP & $51.1 \pm 1.81^{\mathrm{cB} \beta}$ & $54.5 \pm 3.41^{\mathrm{bC} a}$ & $57.2 \pm 2.51^{\mathrm{bD} \alpha}$ & $45.4 \pm 3.91^{\mathrm{bA} \alpha}$ & $50.2 \pm 3.06^{\mathrm{bB} \alpha}$ \\
\hline
\end{tabular}

*: Not normally distributed.

abc: Different letters present significant differences between veneering resin composites within one polymerization device and storage interval.

ABC: Different letters present significant differences between polymerization devices within one veneering resin composite and storage interval.

${ }^{\alpha \beta \gamma}$ : Different letters present significant differences between storage intervals within one polymerization device and one veneering resin composite.

$\left.\eta_{\mathrm{p}}{ }^{2}=0.061\right)$. Therefore, the data were analyzed separately according to the tested hypotheses.

\section{Degree of conversion}

The choice of veneering resin composite showed an influence on DC $(p<0.001-0.042)$, with a majority of groups showing the lowest DC for CI, while NP presented the highest values (Table 2).

Furthermore, an impact of the polymerization device on DC was observed $(p<0.001-0.027)$ : a polymerization with Otoflash G171 resulted in the highest DC for most groups, followed by curing with Laboligth DUO, while the other three polymerization devices showed lower results.

An increase in storage interval led to an increased DC for GP specimens polymerized with bre.Lux Power Unit $(p<0.0085)$, while not presenting an influence on all other groups $(p=0.001-0.626)$.

\section{Martens hardness and elastic indentation modulus}

The choice of veneering resin composite showed an influence on $\mathrm{HM}$ and $\mathrm{E}_{\mathrm{IT}}(p<0.001-0.042)$, with the exception of HM results for specimens polymerized with PCU LED after storage intervals i), ii), and iii), $(p=0.134-0.423)$. NP specimens showed the lowest HM and $\mathrm{E}_{\mathrm{IT}}$ results for most groups, while CI specimens 
Table 3 Martens hardness [N/mm²] and elastic indentation modulus $\left[\mathrm{kN} / \mathrm{mm}^{2}\right]$ for all tested groups

\begin{tabular}{|c|c|c|c|c|c|}
\hline \multirow{2}{*}{$\begin{array}{l}\mathrm{HM} \\
\mathrm{E}_{\mathrm{IT}}\end{array}$} & bre.Lux Power Unit & Labolight DUO & Otoflash G171 & LC-3DPrint Box & PCU LED \\
\hline & Mean \pm SD & Mean \pm SD & Mean \pm SD & Mean \pm SD & Mean \pm SD \\
\hline $\begin{array}{l}\text { i) } \\
\mathrm{NP}\end{array}$ & $\begin{array}{l}233 \pm 18.4^{\mathrm{aA} \alpha} \\
5.92 \pm 0.506^{* \mathrm{aA} \alpha}\end{array}$ & $\begin{array}{l}268 \pm 14.4^{\mathrm{aC} \alpha} \\
6.76 \pm 0.502^{\mathrm{aBC} a}\end{array}$ & $\begin{array}{l}307 \pm 23.3^{\mathrm{aD} \alpha} \\
7.20 \pm 0.700^{\mathrm{aC} \alpha}\end{array}$ & $\begin{array}{l}245 \pm 25.5^{\mathrm{abB} \alpha} \\
5.90 \pm 0.449^{\mathrm{aA} \alpha}\end{array}$ & $\begin{array}{l}255 \pm 44.2^{* \mathrm{aBC} \alpha} \\
6.26 \pm 0.801^{\mathrm{aAB} \alpha}\end{array}$ \\
\hline CI & $\begin{array}{l}296 \pm 77.4^{* \mathrm{cA} \alpha} \\
7.60 \pm 2.26^{* \mathrm{AA} \alpha}\end{array}$ & $\begin{array}{l}367 \pm 27.2^{\mathrm{cB} \alpha} \\
9.53 \pm 0.499^{\mathrm{cB} \alpha}\end{array}$ & $\begin{array}{l}408 \pm 53.8^{* \mathrm{bC} \alpha} \\
10.0 \pm 1.13^{\mathrm{cC} \alpha}\end{array}$ & $\begin{array}{l}267 \pm 63.4^{\mathrm{bA} \alpha} \\
7.40 \pm 1.33^{* \mathrm{bA} \alpha}\end{array}$ & $\begin{array}{l}269 \pm 51.9^{* \mathrm{aA} \alpha} \\
7.67 \pm 1.22^{* \mathrm{bA} \alpha}\end{array}$ \\
\hline GP & $\begin{array}{l}264 \pm 22.6^{* \mathrm{bB} \alpha} \\
6.98 \pm 0.449^{\mathrm{bB} \alpha}\end{array}$ & $\begin{array}{l}296 \pm 30.4^{\mathrm{bC} \alpha} \\
7.69 \pm 0.588^{\mathrm{bC} \alpha}\end{array}$ & $\begin{array}{l}318 \pm 29.7^{\mathrm{aC} \alpha} \\
7.89 \pm 0.637^{\mathrm{bC} \alpha}\end{array}$ & $\begin{array}{l}222 \pm 39.0^{* \mathrm{aA} \alpha} \\
5.58 \pm 1.02^{* \mathrm{aA} \alpha}\end{array}$ & $\begin{array}{l}250 \pm 45.2^{\mathrm{aAB} \alpha} \\
6.68 \pm 0.935^{\mathrm{aAB} \alpha}\end{array}$ \\
\hline $\begin{array}{l}\text { ii) } \\
\text { NP }\end{array}$ & $\begin{array}{l}254 \pm 16.7^{\text {аАа } \beta} \\
6.30 \pm 0.372^{\text {аА } \alpha \beta}\end{array}$ & $\begin{array}{l}284 \pm 12.3^{\mathrm{aB} \alpha} \\
7.24 \pm 0.484^{\mathrm{a} \alpha \beta}\end{array}$ & $\begin{array}{l}301 \pm 25.1^{\mathrm{aC} \alpha} \\
7.15 \pm 0.624^{\mathrm{aB} \alpha}\end{array}$ & $\begin{array}{l}255 \pm 12.5^{* \text { Аа } \alpha \beta} \\
6.18 \pm 0.402^{\text {аА } \alpha \beta}\end{array}$ & $\begin{array}{l}256 \pm 34.9^{\mathrm{aA} \alpha} \\
6.19 \pm 0.546^{* \mathrm{aA} \alpha}\end{array}$ \\
\hline CI & $\begin{array}{l}323 \pm 47.2^{* \mathrm{cB} \alpha \beta} \\
8.28 \pm 1.41^{* \mathrm{cA} \alpha \beta}\end{array}$ & $\begin{array}{l}368 \pm 19.0^{\mathrm{cC} \alpha} \\
9.60 \pm 0.562^{\mathrm{cB} \alpha}\end{array}$ & $\begin{array}{l}418 \pm 36.4^{\mathrm{bD} \alpha} \\
10.3 \pm 0.860^{\mathrm{cC} \alpha}\end{array}$ & $\begin{array}{l}292 \pm 62.2^{\text {bАВ } \alpha \beta} \\
8.19 \pm 1.26^{\text {bАa }}\end{array}$ & $\begin{array}{l}280 \pm 47.0^{\text {сАа } а} \\
8.07 \pm 0.619^{\text {аА } а \beta}\end{array}$ \\
\hline GP & $\begin{array}{l}285 \pm 32.9 * b \mathrm{~s} \alpha \beta \\
7.50 \pm 0.568^{* \mathrm{bB} \alpha \beta}\end{array}$ & $\begin{array}{l}324 \pm 26.3^{\mathrm{bC} \alpha} \\
8.34 \pm 0.529^{\mathrm{bC} \alpha \beta}\end{array}$ & $\begin{array}{l}314 \pm 44.2^{\mathrm{aC} \alpha} \\
7.94 \pm 0.744^{\mathrm{bBC} \alpha}\end{array}$ & $\begin{array}{l}232 \pm 39.2^{\mathrm{aA \alpha}} \\
6.08 \pm 0.875^{\mathrm{aA} \alpha \beta}\end{array}$ & $\begin{array}{l}251 \pm 49.7^{\mathrm{aAB} \alpha} \\
6.78 \pm 1.22^{\mathrm{aA} \alpha}\end{array}$ \\
\hline $\begin{array}{l}\text { iii) } \\
\text { NP }\end{array}$ & $\begin{array}{l}266 \pm 19.7^{* \mathrm{\alpha} \alpha \beta \gamma \gamma} \\
6.90 \pm 0.732^{\text {aBC } \alpha \beta \gamma}\end{array}$ & $\begin{array}{l}289 \pm 24.6^{\mathrm{aB} \alpha} \\
7.33 \pm 0.770^{\mathrm{aC} \alpha \beta}\end{array}$ & $\begin{array}{l}310 \pm 34.3^{\mathrm{aB} \alpha} \\
7.19 \pm 0.957^{\mathrm{aBC} \alpha}\end{array}$ & 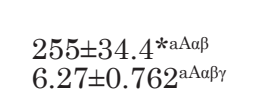 & $\begin{array}{l}286 \pm 18.9^{* \mathrm{aB} \alpha} \\
6.70 \pm 0.442^{\mathrm{aAB} \alpha}\end{array}$ \\
\hline CI & $\begin{array}{l}356 \pm 44.9^{\mathrm{b} \mathrm{\alpha} \beta} \\
9.48 \pm 0.859^{\mathrm{bA \alpha} \beta}\end{array}$ & $\begin{array}{l}386 \pm 28.9^{\mathrm{cB} \alpha} \\
10.1 \pm 0.644^{\mathrm{cA} \alpha}\end{array}$ & $\begin{array}{l}378 \pm 89.0^{* \mathrm{bB} \alpha} \\
9.41 \pm 2.04^{* \mathrm{cA} \alpha}\end{array}$ & $\begin{array}{l}303 \pm 68.4^{* \text { bА } а \beta} \\
9.11 \pm 1.10^{\text {bАaß }}\end{array}$ & 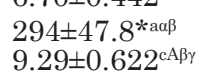 \\
\hline GP & $\begin{array}{l}323 \pm 50.4^{* \mathrm{bBC} \beta} \\
9.02 \pm 0.890^{\mathrm{bC} \gamma}\end{array}$ & $\begin{array}{l}342 \pm 34.3^{\mathrm{bC} \alpha} \\
8.89 \pm 0.658^{\mathrm{bC} \beta}\end{array}$ & $\begin{array}{l}334 \pm 38.0^{\mathrm{aC} \alpha} \\
8.43 \pm 0.755^{\mathrm{bBC} \alpha}\end{array}$ & $\begin{array}{l}247 \pm 40.7^{\mathrm{aA} \alpha} \\
6.46 \pm 1.08^{\mathrm{aA} \alpha \beta}\end{array}$ & $\begin{array}{l}295 \pm 33.8^{\mathrm{aB} \alpha} \\
8.18 \pm 0.448^{\mathrm{bA \alpha} \beta}\end{array}$ \\
\hline $\begin{array}{l}\text { iv) } \\
\mathrm{NP}\end{array}$ & $\begin{array}{l}277 \pm 20.5^{\mathrm{aA} \beta \gamma} \\
7.26 \pm 0.641^{\mathrm{aBC} \beta \gamma}\end{array}$ & $\begin{array}{l}291 \pm 22.6^{\mathrm{aB} \alpha} \\
7.53 \pm 0.466^{* \mathrm{aC} \alpha \beta \gamma}\end{array}$ & $\begin{array}{l}309 \pm 28.5^{* \mathrm{aC} \alpha} \\
7.24 \pm 0.725^{\mathrm{aABC} \alpha}\end{array}$ & $\begin{array}{l}283 \pm 27.8^{\mathrm{aAB} а \beta} \\
6.86 \pm 0.573^{\mathrm{aAB} \beta \gamma \delta}\end{array}$ & $\begin{array}{l}285 \pm 24.3^{\mathrm{aA} \alpha} \\
6.77 \pm 0.582^{\mathrm{aA} \alpha}\end{array}$ \\
\hline CI & $\begin{array}{l}405 \pm 87.1^{\mathrm{cAB \beta}} \\
10.5 \pm 1.86^{\mathrm{cA} \beta}\end{array}$ & $\begin{array}{l}383 \pm 55.2^{\mathrm{cBC} \alpha} \\
10.5 \pm 1.13^{\mathrm{cA} \alpha}\end{array}$ & $\begin{array}{l}404 \pm 47.8^{\mathrm{cBC} \alpha} \\
10.5 \pm 1.10^{\mathrm{cA \alpha} \alpha}\end{array}$ & $\begin{array}{l}340 \pm 69.1^{* \mathrm{bA \alpha} \alpha} \\
9.40 \pm 1.27^{* \mathrm{bA \alpha} \beta}\end{array}$ & $\begin{array}{l}350 \pm 53.6^{* \mathrm{bA \beta}} \\
9.91 \pm 0.820^{\mathrm{cA} \gamma}\end{array}$ \\
\hline GP & $\begin{array}{l}321 \pm 49.1^{\mathrm{bB} \beta} \\
8.48 \pm 1.00^{\mathrm{bA} \beta \gamma}\end{array}$ & $\begin{array}{l}346 \pm 32.6^{\mathrm{bB} \alpha} \\
8.78 \pm 0.778^{\mathrm{bA} \beta}\end{array}$ & $\begin{array}{l}351 \pm 30.1^{\mathrm{bB} \alpha} \\
8.80 \pm 0.880^{* \mathrm{bA} \alpha}\end{array}$ & $\begin{array}{l}348 \pm 66.3^{\mathrm{bB} \beta} \\
8.72 \pm 1.19^{* \mathrm{bA} \gamma}\end{array}$ & $\begin{array}{l}270 \pm 62.6^{\mathrm{aA \alpha}} \\
7.73 \pm 1.00^{\mathrm{bA} \alpha \beta}\end{array}$ \\
\hline $\begin{array}{l}\text { v) } \\
\text { NP }\end{array}$ & $\begin{array}{l}274 \pm 28.6^{\mathrm{aA \beta \gamma \gamma}} \\
7.09 \pm 0.759_{\mathrm{aABC}}^{\alpha \beta \gamma}\end{array}$ & $\begin{array}{l}294 \pm 27.0^{* \mathrm{aA} \alpha} \\
7.62 \pm 0.528^{\mathrm{aC} \alpha \beta \gamma}\end{array}$ & $\begin{array}{l}308 \pm 42.9^{\mathrm{aA} \alpha} \\
7.39 \pm 0.841^{\mathrm{aBC} \alpha}\end{array}$ & $\begin{array}{l}282 \pm 27.7^{\text {аА } \alpha \beta} \\
6.89 \pm 0.688^{\text {aАВ } \beta \gamma \delta}\end{array}$ & $\begin{array}{l}266 \pm 60.7^{\mathrm{aA} \alpha} \\
6.59 \pm 0.980^{\mathrm{aA} \alpha}\end{array}$ \\
\hline CI & $\begin{array}{l}364 \pm 35.3^{\mathrm{cBC} \alpha \beta} \\
9.88 \pm 0.749^{\mathrm{cB} \alpha \beta}\end{array}$ & $\begin{array}{l}395 \pm 54.3^{* \mathrm{cD} \alpha} \\
10.3 \pm 0.840^{* \mathrm{cB} \alpha}\end{array}$ & $\begin{array}{l}406 \pm 60.3^{* \mathrm{bD} \alpha} \\
10.1 \pm 0.853^{\mathrm{cAB} \alpha}\end{array}$ & $\begin{array}{l}313 \pm 66.0^{\text {aАa } \beta} \\
8.73 \pm 1.37^{* \text { bА } \beta}\end{array}$ & $\begin{array}{l}350 \pm 52.3^{\mathrm{bAB \beta}} \\
9.75 \pm 0.899^{\mathrm{cAB} \gamma}\end{array}$ \\
\hline GP & $\begin{array}{l}324 \pm 13.5^{\mathrm{bB} \beta} \\
8.65 \pm 0.443^{\mathrm{bC} \beta \gamma}\end{array}$ & $\begin{array}{l}352 \pm 26.2^{\mathrm{bC} \alpha} \\
9.09 \pm 0.481^{\mathrm{bD} \beta}\end{array}$ & $\begin{array}{l}338 \pm 51.8^{\mathrm{aBC} \alpha} \\
8.48 \pm 0.927^{\mathrm{bCD} \alpha}\end{array}$ & $\begin{array}{l}266 \pm 37.2^{\mathrm{aA} \alpha} \\
7.12 \pm 0.775^{\mathrm{aA} \beta}\end{array}$ & $\begin{array}{l}268 \pm 61.6^{\mathrm{aA} \alpha} \\
7.76 \pm 1.06^{* \mathrm{BB} \alpha \beta}\end{array}$ \\
\hline $\begin{array}{l}\text { vi) } \\
\text { NP }\end{array}$ & $\begin{array}{l}276 \pm 27.2^{\mathrm{aA} \beta \gamma} \\
7.65 \pm 0.719^{\mathrm{aB} \gamma}\end{array}$ & $\begin{array}{l}295 \pm 20.9^{* \mathrm{aB} \alpha} \\
7.98 \pm 0.387^{\mathrm{aB} \beta \gamma}\end{array}$ & $\begin{array}{l}315 \pm 34.8^{* a \mathrm{aC} \alpha} \\
7.49 \pm 0.950^{* \mathrm{aB} \alpha}\end{array}$ & $\begin{array}{l}270 \pm 29.7^{\mathrm{aA} \alpha \beta} \\
6.76 \pm 0.565^{\mathrm{aA} \alpha \beta \gamma \delta}\end{array}$ & $\begin{array}{l}287 \pm 40.9^{\mathrm{aAB} \alpha} \\
6.95 \pm 0.660^{\mathrm{aA} \alpha}\end{array}$ \\
\hline CI & $\begin{array}{l}418 \pm 88.2^{* \mathrm{cBC} \beta} \\
9.81 \pm 1.60^{\operatorname{cA} \beta}\end{array}$ & $\begin{array}{l}437 \pm 97.7^{\mathrm{cBC} \alpha} \\
10.0 \pm 1.38^{* \mathrm{cA} \alpha}\end{array}$ & $\begin{array}{l}423 \pm 59.2^{\mathrm{bC} \alpha} \\
9.63 \pm 0.839^{\mathrm{cA} \alpha}\end{array}$ & $\begin{array}{l}359 \pm 91.0^{\mathrm{bAB} \beta} \\
9.73 \pm 1.72^{\mathrm{bA \beta}}\end{array}$ & $\begin{array}{l}353 \pm 32.8^{\mathrm{bA \beta}} \\
10.1 \pm 0.822^{\mathrm{cA \gamma}}\end{array}$ \\
\hline GP & $\begin{array}{l}319 \pm 39.1^{* \mathrm{bB} \beta} \\
8.63 \pm 0.740^{* \mathrm{bBC} \beta \gamma}\end{array}$ & $\begin{array}{l}342 \pm 30.2^{\mathrm{bB} \alpha} \\
8.99 \pm 0.654^{\mathrm{bC \beta}}\end{array}$ & $\begin{array}{l}342 \pm 33.0^{\mathrm{aB} \alpha} \\
8.80 \pm 0.892^{\mathrm{bC} \alpha}\end{array}$ & $\begin{array}{l}256 \pm 31.4^{\mathrm{aA \alpha}} \\
6.98 \pm 0.664^{\text {аА } \alpha \beta}\end{array}$ & $\begin{array}{l}272 \pm 50.4^{\mathrm{aA} \alpha} \\
7.92 \pm 0.967^{\mathrm{bB} \alpha \beta}\end{array}$ \\
\hline $\begin{array}{l}\text { vii) } \\
\text { NP }\end{array}$ & $\begin{array}{l}291 \pm 25.9^{* \mathrm{aB} \gamma} \\
7.56 \pm 1.13^{\mathrm{aBC} \gamma}\end{array}$ & $\begin{array}{l}290 \pm 42.3^{* \mathrm{aB} \alpha} \\
8.34 \pm 0.734^{* \mathrm{aD} \gamma}\end{array}$ & $\begin{array}{l}335 \pm 18.0^{\mathrm{aC} \alpha} \\
7.81 \pm 0.681^{\mathrm{aC} \alpha}\end{array}$ & $\begin{array}{l}284 \pm 26.3^{\mathrm{aAB} \alpha \beta} \\
7.08 \pm 0.503^{\mathrm{aAB} \gamma \delta}\end{array}$ & $\begin{array}{l}281 \pm 40.0^{* \mathrm{aA} \alpha} \\
6.85 \pm 0.856^{\mathrm{aA} \alpha}\end{array}$ \\
\hline CI & $\begin{array}{l}349 \pm 55.7^{\mathrm{bA \alpha \beta}} \\
9.49 \pm 1.56^{\text {* } \mathrm{bAB} \alpha \beta}\end{array}$ & $\begin{array}{l}394 \pm 49.6^{\mathrm{cB} \alpha} \\
10.4 \pm 1.15^{\mathrm{cBC} \alpha}\end{array}$ & $\begin{array}{l}434 \pm 53.9^{* \mathrm{bC} \alpha} \\
10.7 \pm 1.10^{\mathrm{cC} \alpha}\end{array}$ & $\begin{array}{l}319 \pm 59.6^{\text {аА } \alpha \beta} \\
8.92 \pm 1.29^{\text {bА } \alpha \beta}\end{array}$ & $\begin{array}{l}336 \pm 44.4^{\mathrm{bAa} \beta} \\
9.51 \pm 0.891^{\mathrm{cA} \gamma}\end{array}$ \\
\hline GP & $\begin{array}{l}310 \pm 47.9^{* \mathrm{aBC} \alpha \beta} \\
8.23 \pm 1.10^{\mathrm{aB} \beta \gamma}\end{array}$ & $\begin{array}{l}350 \pm 38.4^{* \mathrm{bD} \alpha} \\
9.08 \pm 0.981^{\mathrm{bC} \beta}\end{array}$ & $\begin{array}{l}341 \pm 44.6^{\mathrm{aCD} \alpha} \\
8.57 \pm 0.745^{\mathrm{bBC} \alpha}\end{array}$ & $\begin{array}{l}271 \pm 32.1^{\mathrm{aA} \alpha} \\
7.40 \pm 0.638^{\mathrm{aA} \beta \gamma}\end{array}$ & $\begin{array}{l}296 \pm 45.8 \mathrm{a}^{\mathrm{bAB} \alpha} \\
8.41 \pm 0.742^{\mathrm{bB \beta}}\end{array}$ \\
\hline $\begin{array}{l}\text { viii) } \\
\mathrm{NP}\end{array}$ & $\begin{array}{l}278 \pm 27.3^{\mathrm{aA} \beta \gamma} \\
7.37 \pm 0.961^{\mathrm{aABC} \beta \gamma}\end{array}$ & $\begin{array}{l}301 \pm 20.0^{\mathrm{aBC} \alpha} \\
7.87 \pm 0.541^{* \mathrm{aC} \beta \gamma}\end{array}$ & $\begin{array}{l}309 \pm 31.0^{* \mathrm{aC} \alpha} \\
7.42 \pm 0.733^{\mathrm{aBC} \alpha}\end{array}$ & $\begin{array}{l}295 \pm 23.4^{\mathrm{bABC} \beta} \\
7.40 \pm 0.468^{\mathrm{aBC} \delta}\end{array}$ & $\begin{array}{l}275 \pm 45.3^{\mathrm{aAB} \alpha} \\
6.70 \pm 1.10^{\mathrm{aA} \alpha}\end{array}$ \\
\hline CI & $\begin{array}{l}370 \pm 33.1^{\text {cAa } \beta} \\
10.3 \pm 0.899^{\text {cA } \beta}\end{array}$ & $\begin{array}{l}383 \pm 60.9^{\mathrm{bA} \alpha} \\
10.2 \pm 1.33^{\mathrm{cA} \alpha}\end{array}$ & $\begin{array}{l}405 \pm 91.7^{* \mathrm{cA} \alpha} \\
10.0 \pm 2.02^{* \mathrm{cA} \alpha}\end{array}$ & $\begin{array}{l}353 \pm 93.6^{\mathrm{cA \alpha} \beta} \\
9.63 \pm 1.47^{\mathrm{bA \beta}}\end{array}$ & $\begin{array}{l}347 \pm 47.3^{\mathrm{bA \beta}} \\
10.1 \pm 0.801^{\mathrm{cA} \gamma}\end{array}$ \\
\hline GP & $\begin{array}{l}324 \pm 30.3^{\mathrm{bBC \beta}} \\
8.66 \pm 0.582^{\mathrm{bBC \beta \gamma}}\end{array}$ & $\begin{array}{l}346 \pm 43.2^{\mathrm{bCD} \alpha} \\
9.17 \pm 0.675^{\mathrm{bC} \beta}\end{array}$ & $\begin{array}{l}352 \pm 21.9^{\mathrm{bD} \alpha} \\
8.94 \pm 0.411^{\mathrm{bBC} \alpha}\end{array}$ & $\begin{array}{l}267 \pm 34.4^{\mathrm{aA \alpha} \alpha} \\
7.32 \pm 0.934^{\mathrm{aA \beta \gamma}}\end{array}$ & $\begin{array}{l}289 \pm 52.4^{\mathrm{aAB} \alpha} \\
8.16 \pm 1.43^{* \mathrm{bB} \alpha \beta}\end{array}$ \\
\hline
\end{tabular}

*: Not normally distributed.

abc: Different letters present significant differences between veneering resin composites within one polymerization device and storage interval.

ABC: Different letters present significant differences between polymerization devices within one veneering resin composite and storage interval.

${ }^{\alpha \beta \gamma}$ : Different letters present significant differences between storage intervals within one polymerization device and one veneering resin composite. 
Table 4 Flexural strength [MPa] and Weibull moduli for all tested groups

\begin{tabular}{|c|c|c|c|c|c|}
\hline \multirow[t]{2}{*}{ FS } & bre.Lux Power Unit & Labolight DUO & Otoflash G171 & LC-3DPrint Box & PCU LED \\
\hline & Mean \pm SD & Mean \pm SD & Mean \pm SD & Mean \pm SD & Mean \pm SD \\
\hline $\mathrm{NP}$ & $\begin{array}{l}90.1 \pm 17.5^{\mathrm{aB}} \\
5.50^{\mathrm{bA}}\end{array}$ & $\begin{array}{l}90.0 \pm 19.0^{* \mathrm{bB}} \\
5.02^{\mathrm{aA}}\end{array}$ & $\begin{array}{l}95.3 \pm 16.3^{\mathrm{bB}} \\
6.08^{\mathrm{aA}}\end{array}$ & $\begin{array}{l}85.0 \pm 19.4^{\mathrm{aB}} \\
4.44^{\mathrm{aA}}\end{array}$ & $\begin{array}{l}66.0 \pm 15.2^{* a \mathrm{~A}} \\
4.25^{\mathrm{abA}}\end{array}$ \\
\hline CI & $\begin{array}{l}99.8 \pm 35.3^{\mathrm{aA}} \\
2.96^{\mathrm{aA}}\end{array}$ & $\begin{array}{l}136 \pm 42.8^{\mathrm{cC}} \\
3.41^{\mathrm{aA}}\end{array}$ & $\begin{array}{l}128 \pm 30.1^{\mathrm{cBC}} \\
4.26^{\mathrm{aA}}\end{array}$ & $\begin{array}{l}87.1 \pm 25.0^{\mathrm{aA}} \\
4.21^{\mathrm{aA}}\end{array}$ & $\begin{array}{l}103 \pm 41.1^{\mathrm{bAB}} \\
2.84^{\mathrm{aA}}\end{array}$ \\
\hline GP & $\begin{array}{l}78.7 \pm 22.7^{\mathrm{aA}} \\
3.89^{\mathrm{abA}}\end{array}$ & $\begin{array}{l}71.1 \pm 24.5^{\mathrm{aA}} \\
3.27^{\mathrm{aA}}\end{array}$ & $\begin{array}{l}71.3 \pm 19.8^{\mathrm{aA}} \\
4.31^{\mathrm{aAB}}\end{array}$ & $\begin{array}{l}75.0 \pm 7.83^{\mathrm{aA}} \\
12.4^{\mathrm{bC}}\end{array}$ & $\begin{array}{l}76.1 \pm 11.3^{\mathrm{abA}} \\
7.46^{\mathrm{bBC}}\end{array}$ \\
\hline
\end{tabular}

*: Not normally distributed.

abc: Different letters present significant differences between veneering resin composites within one polymerization device.

ABC: Different letters present significant differences between polymerization devices within one veneering resin composite.

presented the highest values (Table 3).

An impact of the polymerization device was also observed ( $p<0.001-0.027)$, except for HM results of NP specimens after storage interval v), CI specimens after storage interval viii) and $\mathrm{E}_{\mathrm{IT}}$ results for $\mathrm{CI}$ after storage intervals iii), iv), vi), viii) and GP after storage interval iv), $(p=0.052-0.675)$. A polymerization with Otoflash G171 or Labolight DUO resulted in higher HM and $\mathrm{E}_{\mathrm{IT}}$ results for a majority of groups.

Regarding HM results, storage showed an impact on NP, CI, and GP groups cured with either bre.Lux Power Unit or LC-3DPrint Box and CI specimens polymerized with PCU LED $(p<0.000125)$, while the other groups did not present significant results $(p=0.0021-0.145)$. Storage also presented an influence on $\mathrm{E}_{\mathrm{IT}}$ results of all groups $(p<0.000125)$, except specimens polymerized with Otoflash G171 $(p=0.005-0.089)$, NP specimens cured with PCU LED $(p=0.008)$, and CI specimens polymerized with Labolight DUO $(p=0.014)$. For groups affected by storage, an increase in storage time led to increased $\mathrm{HM}$ and $\mathrm{E}_{\mathrm{IT}}$ values.

\section{Flexural strength and Weibull modulus}

The choice of veneering resin composite did not show an impact on FS for specimens polymerized with bre.Lux Power Unit ( $p=0.238)$ and LC-3DPrint Box $(p=0.253)$. For Labolight DUO $(p=0.001)$ and Otoflash G171 $(p<0.001)$, GP showed the lowest FS, while CI presented the highest results (Fig. 3). NP led to lower FS results than CI within PCU LED polymerized specimens $(p=0.018)$.

When regarding Weibull moduli for specimens polymerized with Laboligth DUO or Otoflash G171, the veneering resin composite did not present an influence. Specimens polymerized with bre.Lux Power Unit did, however, present higher Weibull moduli for NP than for CI, while LC-3DPrint Box presented the highest values for GP. Polymerization with PCU LED furthermore resulted in higher Weibull moduli for GP than CI.

The choice of polymerization device showed an influence on FS within veneering resin composites NP $(p=0.002)$ and CI $(p=0.006)$, but not for GP $(p=0.756)$. PCU LED resulted in lower FS for NP specimens than

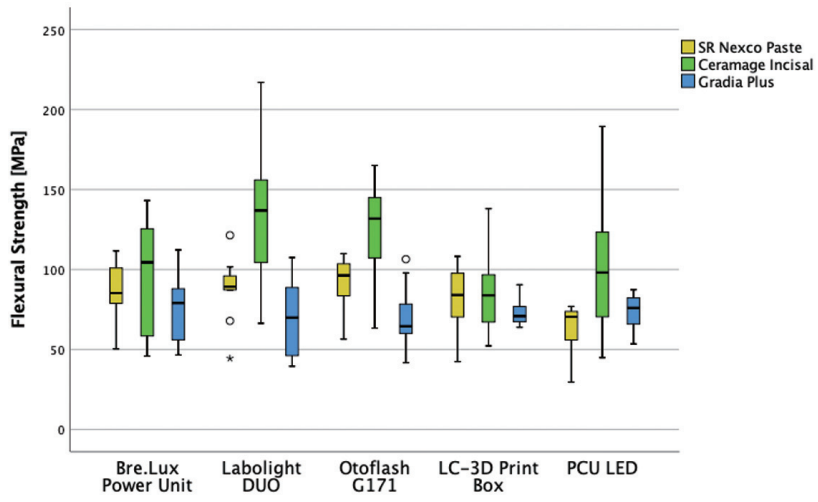

Fig. 3 Flexural strength results of the three veneering resin composites cured with five different polymerization devices.

all other polymerization devices. Within CI, higher FS values were observed for Labolight DUO and Otoflash G171.

In contrast to this finding, the choice of polymerization device did not show an influence on the Weibull modulus for veneering resin composites NP and CI, while a polymerization with LC-3DPrint Box or PCU LED led to a higher Weibull modulus for GP specimens.

\section{DISCUSSION}

The aim of this study was to examine the degree of conversion, Martens hardness, elastic indentation modulus, and flexural strength of three veneering resin composites cured with five polymerization devices after different storage intervals. The tested hypotheses, stating that neither the use of different veneering resin composites, nor the choice of polymerization device or storage interval showed an impact on DC, HM, $\mathrm{E}_{\mathrm{IT}}$, and FS, had to be rejected.

\section{Influence of the resin composite}

While Ceramage Incisal showed the lowest degree of conversion and highest values for Martens hardness and 
elastic indentation modulus, SR Nexco Paste specimens presented the highest degree of conversion paired with the lowest Martens hardness and elastic indentation modulus results. These findings, though contradictory to the assumption that a high degree of conversion goes hand in hand with high mechanical propertie ${ }^{21,22)}$, could be explained by an increased amount of fillers in the microhybrid resin composite Ceramage Incisal. While the filler concentration has been shown to play a prominent role in determining the properties of resin composites, an early investigation found no correlation between the degree of conversion and the mechanical properties of the composite resins ${ }^{23)}$. A high amount of fillers should however entail a low degree of conversion, as the overall presence of monomers which undergo polymerization is reduced. This theory was accentuated by an investigation examining the effect of filler loading on the conversion of photoactivated, resin-based composites with Fourier transform infrared spectroscopy ${ }^{24}$. When regarding the composition of Ceramage Incisal compared with the nanohybrid resin composite SR Nexco Paste, the high amount of zirconium silicate fillers and an increased mass fraction of inorganic fillers are noteworthy and a plausible causal factor for the observed superior mechanical results.

With the choice of veneering resin composite not showing an impact on flexural strength for specimens polymerized with bre.Lux Power Unit and LC-3DPrint Box, it can be concluded that these two polymerization devices lead to comparable results for all three veneering resin composites. For Labolight DUO, Otoflash G171, and PCU LED, Ceramage Incisal presented the highest flexural strength results. It might thus be assumed that the photo initiators found in Ceramage Incisal are ideally matched to the wavelengths and intensities provided by the three aforementioned polymerization devices. As the manufacturers only supply a recommended exposure time with a very limited number of polymerization devices for their respective resin composite, but do not supply the photo initiation systems used in the material's composition, this point cannot be validated. The authors adhered to a polymerization duration of 180 $\mathrm{s}$ for all polymerization devices and resin composites to ensure comparability of the results.

With the Weibull modulus providing an assessment of a material's variability, it can be postulated that veneering resin composite-polymerization device combinations that achieved high results present an optimal match regarding homogeneity and reliability. SR Nexco Paste specimens polymerized with bre.Lux Power Unit and Gradia Plus specimens cured with either LC-3DPrint Box or PCU LED showed significantly higher Weibull moduli and might thus be optimally matched. These observations will have to be confirmed in further investigations.

\section{Influence of the polymerization device}

Polymerization with Otoflash G171 resulted in a high degree of conversion and high results for Martens hardness and elastic indentation modulus, followed by curing with Laboligth DUO, while the other three polymerization devices showed similar lower results. The observed absolute values correspond with the current literature, where the degree of conversion for dimethacrylate polymers has been described to range between $43 \%$ and $75 \%{ }^{25,26)}$. Curing with Otoflash G171 was carried out under nitrogen atmosphere using two flashlight lamps at a broad wavelength spectrum from 280-580 nm. Otoflash G171 does not supply a constant irradiation as seen for a majority of polymerization devices, but repeated concentrated flashlights, which could lead to an increased light intensity resulting in an optimized polymerization and in consequence, a higher degree of conversion and superior mechanical properties. The broad wavelength spectrum ensures photoinitiation for a wide range of initiator systems, while the polymerization under nitrogen atmosphere prevents the formation of an oxygen inhibition layer and thus enables a polymerization of the very surface. One study investigating the impact of post curing on fixed dental prostheses made of resin material already observed increased fracture load results employing Otoflash $\mathrm{G}_{171^{27}}$. When comparing Laboligth DUO to the three less effective polymerization devices, the use of twelve blue and three purple LEDs becomes apparent, producing a shorter violet wavelength for photoinitiators that are sensitive to light from $350-420 \mathrm{~nm}$, and a longer blue wavelength, mainly for camphorquinone at $468 \mathrm{~nm}$, thus ensuring an activation of a wide range of photoinitiators ${ }^{28,29)}$.

The choice of polymerization device showed an influence on flexural strength within veneering resin composites SR Nexco Paste and Ceramage Incisal, but not for Gradia Plus, thus advertising the photo initiation system found in Gradia Plus as a universal system that enables curing with a broad selection of polymerization devices. PCU LED resulted in lower flexural strength for SR Nexco Paste specimens than all other polymerization devices. One notable factor differentiating PCU LED's curing procedure from the others is the generated vacuum atmosphere, which should in theory act in a similar manner as the nitrogen atmosphere in trying to prevent the formation of an oxygen inhibition layer. One study has, however, already shown a highly significant statistical reduction in the degree of conversion for each resin cured under air inhibition conditions ${ }^{30)}$. The authors would furthermore like to point out that the drawing of the vacuum, which takes place prior to curing, might influence the layering and form of the still flexible resin composite. This property could limit its application to only include specimens that are firmly compressed and fixated during polymerization. While this set-up was possible in the present investigation, a broad implementation of this approach in dental laboratories only appears to be feasible in a two-step curing process: 1 ) without vacuum to ensure the resin composite's form and 2) with vacuum to enhance the curing process. Further studies are warranted to investigate the influence of a vacuum atmosphere during polymerization on the mechanical properties of resin 
composites. Within Ceramage Incisal specimens, higher flexural strength values were observed for Labolight DUO and Otoflash G171. These findings are in line with the aforementioned and discussed observations regarding the superior results achieved with these two polymerization devices.

\section{Influence of storage}

An increase in storage led to an increased degree of conversion for Gradia Plus specimens polymerized with bre.Lux Power Unit, while not presenting an influence on all other groups. This finding indicates that while the four other polymerization devices led to an immediate and complete conversion of available double to single bonds, the combination of curing with bre.Lux Power Unit and the material properties of resin composite Gradia Plus gained a benefit from a prolonged storage interval. An increase in the degree of conversion over time has been observed in several investigations ${ }^{15,31,32)}$. Furthermore, a release of residual monomers from denture materials for up to 38 months $^{33)}$ suggests, that the polymerization reaction taking place might not be terminated by the extinguishing of the light curing device, but that residual radicals can convert double bonds over a prolonged time period, thus leading to an increased degree of conversion. A resin composite's biocompatibility could thus benefit by storage, for example in water at $37^{\circ} \mathrm{C}$ for $24 \mathrm{~h}$, a procedure that is widespread in the processing of dentures made from acrylic resins ${ }^{34}$. Another investigation examining cytotoxic effects of dental composites found a 7 days incubation under cell culture conditions to diminish a composite's cytotoxicity ${ }^{35}$. With the relationship between the degree of conversion and residual monomer elution being a controversial topic ${ }^{36,37)}$, this research area requires a further investigation of both parameters over time, with specimens stored for example in water or artificial saliva.

Storage showed an impact on Martens hardness for groups cured with either bre.Lux Power Unit or LC-3DPrint Box and Ceramage Incisal specimens polymerized with PCU LED, while the other groups did not present significant results. This result matches previous findings, where a study investigating the effect of aging on the Martens hardness of four different resin composites did not find a significant correlation ${ }^{38}$. The observed result furthermore once again indicates that while a polymerization with Otoflash G171 or Laboligth DUO led to an immediate and total conversion of available double to single bonds, underlining the superior results achieved by a polymerization with those two devices mentioned and discussed in detail above, a polymerization with bre.Lux Power Unit or LC-3DPrint Box and the combination of a curing with PCU LED and Ceramage Incisal's material properties gained a benefit from an increased storage time.

Storage also presented an influence on the elastic indentation modulus of all groups except specimens polymerized with Otoflash G171, SR Nexco Paste specimens cured with PCU LED and Ceramage Incisal specimens polymerized with Labolight DUO. These results emphasize the optimal conversion obtained for all three veneering resin composites by curing with Otoflash G171, while SR Nexco Paste specimens seem to be ideally suited for a polymerization with PCU LED, potentially profiting from a polymerization under vacuum, while Ceramage Incisal specimens might have benefited by the wide wavelength range obtained by a curing with Labolight DUO.

For groups that were affected by storage, an increase in storage time led to an overall increase in Martens hardness and elastic indentation modulus values. This outcome stands in contrast to the observed results regarding the degree of conversion, as increased values for the two mechanical parameters indicate a further curing process taking place, which can only be possible by a further conversion of double to single bonds. In consequence, the influence of storage should correlate for all three tested parameters and affect the same groups.

As this novel investigation entails several limitations, such as a restricted number of examined resin composite materials and polymerization devices, the present findings should be investigated in further in vitro and in vivo studies. Polymerization might be negatively influenced by the manufacturing process that includes polymerization through a glass microscope slide, which could affect the wavelengths and light intensities of the employed polymerization devices and subsequent polymerization of the resin composite materials. In addition, a possible influence of storage on the flexural strength of the tested materials should be examined. As the measurement set-up for biaxial flexural strength is designed for brittle materials, its reliability for materials showing plastic deformations must be critically evaluated with factors such as deformation and strain rate having to be taken into account. While Raman spectra enable an assessment of a material's composition at one point in a material's diameter, the degree of conversion at other points, for example $0.75 \mathrm{~mm}$ underneath the surface, might deviate. The waist and depth of focus of the focused laser beam depend on the numerical aperture of the microscope objective and extent to which its entrance pupil is filled ${ }^{39}$. While the approximate depth of field can be calculated ${ }^{40)}$, it remains unclear to what extent this parameter might be influenced by the manufacturing process or subsequent polishing, which is routinely carried out to improve surface quality and which could bias the Raman measurement if the depth of focus is too close to the surface. For resin composites comprising a high amount of monomers without aromatic $\mathrm{C}=\mathrm{C}$ double bonds, the peak at $1,610 \mathrm{~cm}^{-1}$, which might also be attributed to a N-H deformation, can present a low intensity. In such cases, the use of other internal standard peaks, such as the peak at 1,715 $\mathrm{cm}^{-1}$, corresponding to the carbonyl double bond, could present a higher reliability and reproducibility. While the authors observed consistent results for the reference band at $1,610 \mathrm{~cm}^{-1}$ in the present study, the origin of this band is controversial, as the manufacturers did not specify all tested materials to possess aromatic 
$\mathrm{C}=\mathrm{C}$ double bonds. Future studies employing nuclear magnetic resonance spectroscopy could provide valuable insights into the employed methodology by identifying monomolecular organic compounds. As the potential fluorescence of coloring agents could interfere with Raman scattering, the wavelength of the excitation light should be varied in future preliminary tests to allow for a differentiation between the two effects. Whether an increased degree of conversion enhances biocompatibility and correlates with the elution of residual monomers remains controversial ${ }^{36,37}$, with the filler content playing a crucial role. Employing a Raman spectrophotometer to determine a composite's degree of conversion should thus be critically evaluated by comparing this technique with tried and tested practices such as gas chromatography/ mass spectrometry in the future.

\section{CONCLUSIONS}

Within the limitations of this study, the following conclusions can be drawn:

1. Polymerization with Otoflash G171 and Labolight DUO led to increased degree of conversion, Martens hardness, and elastic indentation modulus results.

2. Ceramage Incisal presented the lowest degree of conversion and highest Martens hardness and elastic indentation modulus values, while SR Nexco Paste showed the highest degree of conversion and lowest Martens hardness and elastic indentation modulus results.

3. Within groups polymerized with Otoflash G171, Laboligth DUO and PCU LED, the highest flexural strength was observed for Ceramage Incisal.

4. Storage showed no influence on the degree of conversion, Martens hardness and elastic indentation modulus of specimens cured with Otoflash G171 or Labolight DUO.

\section{ACKNOWLEDGMENTS}

This research was supported by the German Research Foundation DFG (INST 409/190-1 FUGG contract) in the field of Raman spectrometry.

\section{REFERENCES}

1) Ilie N, Hickel R. Resin composite restorative materials. Aust Dent J 2011; 56 Suppl 1: 59-66.

2) Yadav RD, Raisingani D, Jindal D, Mathur R. A comparative analysis of different finishing and polishing devices on nanofilled, microfilled, and hybrid composite: A scanning electron microscopy and profilometric study. Int $\mathrm{J}$ Clin Pediatr Dent 2016; 9: 201-208.

3) Beun S, Glorieux T, Devaux J, Vreven J, Leloup G. Characterization of nanofilled compared to universal and microfilled composites. Dent Mater 2007; 23: 51-59.

4) Alzraikat H, Burrow MF, Maghaireh GA, Taha NA. Nanofilled resin composite properties and clinical performance: A review. Oper Dent 2018; 43: E173-E190.

5) Borba M, Della Bona A, Cecchetti D. Flexural strength and hardness of direct and indirect composites. Braz Oral Res 2009; 23: 5-10.

6) Alves PB, Brandt WC, Neves AC, Cunha LG, Silva-Concilio LR. Mechanical properties of direct and indirect composites after storage for $24 \mathrm{~h}$ and 10 months. Eur J Dent 2013; 7: 117-122.

7) Ferracane JL. Elution of leachable components from composites. J Oral Rehabil 1994; 21: 441-452.

8) dos Santos RL, de Sampaio GA, de Carvalho FG, Pithon MM, Guenes GM, Alves PM. Influence of degree of conversion on the biocompatibility of different composites in vivo. J Adhes Dent 2014; 16: 15-20.

9) Ikemura K, Endo T. A review of the development of radical photopolymerization initiators used for designing light-curing dental adhesives and resin composites. Dent Mater J 2010; 29: 481-501.

10) Santini A, Gallegos IT, Felix CM. Photoinitiators in dentistry: a review. Prim Dent J 2013; 2: 30-33.

11) Mousavinasab SM. Biocompatibility of composite resins. Dent Res J (Isfahan) 2011; 8: S21-29.

12) Bettencourt AF, Neves CB, de Almeida MS, Pinheiro LM, Oliveira SA, Lopes LP, et al. Biodegradation of acrylic based resins: A review. Dent Mater 2010; 26: e171-180.

13) Tsuda H, Arends J. Raman spectroscopy in dental research: a short review of recent studies. Adv Dent Res 1997; 11: 539547.

14) Pianelli C, Devaux J, Bebelman S, Leloup G. The microRaman spectroscopy, a useful tool to determine the degree of conversion of light-activated composite resins. J Biomed Mater Res 1999; 48: 675-681.

15) Mohamad D, Young RJ, Mann AB, Watts DC. Postpolymerization of dental resin composite evaluated with nanoindentation and micro-Raman spectroscopy. Arch Orofac Sci 2007; 2: 26-31.

16) Galvao MR, Caldas SG, Bagnato VS, de Souza Rastelli AN, de Andrade MF. Evaluation of degree of conversion and hardness of dental composites photo-activated with different light guide tips. Eur J Dent 2013; 7: 86-93.

17) DINISO 14577. Metallic materials - Instrumented indentation test for hardness and materials parameters - Part 1: Test method (ISO 14577-1:2015) 2015.

18) Greaves GN, Greer AL, Lakes RS, Rouxel T. Poisson's ratio and modern materials. Nat Mater 2011; 10: 823-837.

19) DIN ISO 6872. Dentistry —Ceramic materials 2008.

20) Butikofer L, Stawarczyk B, Roos M. Two regression methods for estimation of a two-parameter Weibull distribution for reliability of dental materials. Dent Mater 2015; 31: e33-50.

21) Calheiros FC, Daronch M, Rueggeberg FA, Braga RR. Degree of conversion and mechanical properties of a BisGMA:TEGDMA composite as a function of the applied radiant exposure. J Biomed Mater Res B Appl Biomater 2008; 84: 503-509.

22) Peutzfeldt A. Resin composites in dentistry: the monomer systems. Eur J Oral Sci 1997; 105: 97-116.

23) Chung KH, Greener EH. Correlation between degree of conversion, filler concentration and mechanical properties of posterior composite resins. J Oral Rehabil 1990; 17: 487-494.

24) Halvorson RH, Erickson RL, Davidson CL. The effect of filler and silane content on conversion of resin-based composite. Dent Mater 2003; 19: 327-333.

25) Imazato S, McCabe JF, Tarumi H, Ehara A, Ebisu S. Degree of conversion of composites measured by DTA and FTIR. Dent Mater 2001; 17: 178-183.

26) Peutzfeldt A, Sahafi A, Asmussen E. Characterization of resin composites polymerized with plasma arc curing units. Dent Mater 2000; 16: 330-336.

27) Reymus M, Fabritius R, Kessler A, Hickel R, Edelhoff D, Stawarczyk B. Fracture load of 3D-printed fixed dental prostheses compared with milled and conventionally 
fabricated ones: the impact of resin material, build direction, post-curing, and artificial aging-an in vitro study. Clin Oral Investig 2020; 24: 701-710.

28) Cramer NB, Stansbury JW, Bowman CN. Recent advances and developments in composite dental restorative materials. J Dent Res 2011; 90: 402-416.

29) Rueggeberg FA. State-of-the-art: dental photocuring: a review. Dent Mater 2011; 27: 39-52.

30) Franz A, Konig F, Lucas T, Watts DC, Schedle A. Cytotoxic effects of dental bonding substances as a function of degree of conversion. Dent Mater 2009; 25: 232-239.

31) Ghavami-Lahiji M, Firouzmanesh M, Bagheri H, Jafarzadeh Kashi TS, Razazpour F, Behroozibakhsh M. The effect of thermocycling on the degree of conversion and mechanical properties of a microhybrid dental resin composite. Restor Dent Endod 2018; 43: e26.

32) Tonetto MR, Pinto SC, Rastelli Ade N, Borges AH, Saad JR, Pedro FL, et al. Degree of conversion of polymer-matrix composite assessed by FTIR analysis. J Contemp Dent Pract 2013; 14: 76-79.

33) Zissis A, Yannikakis S, Polyzois G, Harrison A. A long term study on residual monomer release from denture materials. Eur J Prosthodont Restor Dent 2008; 16: 81-84.

34) Bayraktar G, Guvener B, Bural C, Uresin Y. Influence of polymerization method, curing process, and length of time of storage in water on the residual methyl methacrylate content in dental acrylic resins. J Biomed Mater Res B Appl Biomater 2006; 76: 340-345.

35) Franz A, Konig F, Anglmayer M, Rausch-Fan X, Gille G, Rausch WD, et al. Cytotoxic effects of packable and nonpackable dental composites. Dent Mater 2003; 19: 382392.

36) Durner J, Obermaier J, Draenert M, Ilie N. Correlation of the degree of conversion with the amount of elutable substances in nano-hybrid dental composites. Dent Mater 2012; 28: 11461153.

37) Obradvoic-Djuricic Koskova MV, Radisic Marina, Lausevic Mila. Correlation between the degree of conversion and the elution of leachable components from dental resin-based cements. J Serb Chem Soc 2011; 76: 1307-1323.

38) Fischer J, Roeske S, Stawarczyk B, Hammerle CH. Investigations in the correlation between Martens hardness and flexural strength of composite resin restorative materials. Dent Mater J 2010; 29: 188-192.

39) Everall NJ. Confocal Raman microscopy: common errors and artefacts. Analyst 2010; 135: 2512-2522

40) Adar F. Developments in Raman Microanalysis, San Francisco, San Francisco Press, 1981, p. 67. 